\title{
Femtosecond $x$-ray pulse temporal characterization in free-electron lasers using a transverse deflector
}

\author{
Y. Ding, ${ }^{1, *}$ C. Behrens,${ }^{2}$ P. Emma, ${ }^{1}$ J. Frisch, ${ }^{1}$ Z. Huang, ${ }^{1}$ H. Loos, ${ }^{1}$ P. Krejcik,,${ }^{1}$ and M-H. Wang ${ }^{1}$ \\ ${ }^{1}$ SLAC National Accelerator Laboratory, Menlo Park, California 94025, USA \\ ${ }^{2}$ Deutsches Elektronen-Synchrotron (DESY), Hamburg, Germany
}

(Received 18 August 2011; published 7 December 2011)

\begin{abstract}
We propose a novel method to characterize the temporal duration and shape of femtosecond $\mathrm{x}$-ray pulses in a free-electron laser (FEL) by measuring the time-resolved electron-beam energy loss and energy spread induced by the FEL process, with a transverse radio-frequency deflector located after the undulator. Its merits are simplicity, high resolution, wide diagnostic range, and noninvasive to user operation. When the system is applied to the Linac Coherent Light Source, the world's most powerful x-ray FEL, it can provide single-shot measurements of the electron-beam and x-ray pulses with a resolution on the order of 1-2 femtoseconds rms.
\end{abstract}

DOI: 10.1103/PhysRevSTAB.14.120701

PACS numbers: 41.60.Cr, 41.50.+h

\section{INTRODUCTION}

The successful operation of the Linac Coherent Light Source (LCLS) [1], with its capability of generating freeelectron laser (FEL) $x$-ray pulses from a few femtoseconds (fs) up to a few hundred fs, opens up vast opportunities for studying atoms and molecules on this unprecedented ultrashort time scale. However, tremendous challenges remain in the measurement and control of these ultrashort pulses with femtosecond precision, for both the electronbeam (e-beam) and the x-ray pulses.

For ultrashort e-beam bunch length measurements, a standard method has been established at LCLS using an S-band radio-frequency (rf) deflector, which works like a streak camera for electrons and is capable of resolving bunch lengths as short as $\sim 10 \mathrm{fs}$ rms [1]. However, the e-beam with low charges of $20 \mathrm{pC}$ at LCLS, which is expected to be less than $10 \mathrm{fs}$ in duration, is too short to be measured using this transverse deflector [2]. Recently, a new method of measurement that maps time to energy has been proposed [3] and demonstrated [4] at LCLS, with a measured e-beam resolution about $1 \mathrm{fs}$ rms.

The measurement of the electron bunch length is helpful in estimating the FEL $\mathrm{x}$-ray pulse duration. However, for a realistic beam, such as that with a Gaussian shape or a spiky profile, the FEL amplification varies along the bunch due to peak current or emittance variation. This will result in differences between the temporal shape or duration of the electron bunch and the x-ray pulse. Initial experiments at LCLS have revealed that characterization of the $\mathrm{x}$-ray pulse duration on a shot-by-shot basis is critical for the

\footnotetext{
*ding@slac.stanford.edu
}

Published by the American Physical Society under the terms of the Creative Commons Attribution 3.0 License. Further distribution of this work must maintain attribution to the author(s) and the published article's title, journal citation, and DOI. interpretation of the data. However, in the femtosecond regime, conventional photodetectors and streak cameras do not have a fast enough response time for characterizing the ultrashort x-ray pulses. In addition, because of the vanishingly small cross sections in nonlinear processes at x-ray wavelengths, it makes the temporal correlation techniques very difficult to realize. To overcome these difficulties, some new methods have been studied recently. One of the time-domain methods is the terahertz-field-driven $\mathrm{x}$-ray streak camera [5], where a terahertz field is used to modulate the photoelectrons generated from $\mathrm{x}$-ray gas ionization. By measuring the energy distribution of the photoelectrons the x-ray pulse length can be determined. Here the terahertz radiation is generated from the same electron bunch in a dedicated undulator to achieve a synchronization between the x-ray and terahertz fields. An external optical laser has also been tested to streak the photoelectrons at the LCLS, but single-shot measurements are not possible due to the synchronization difficulties [6]. In the frequency domain, pulse length measurements based on statistical analysis of the fluctuations in the radiation spectrum have been reported [7], and recently this technique has been experimentally studied at LCLS from multishot analysis of the spectral correlation function [8]. There are also other techniques proposed or tested, such as X-ray autocorrelation or x-ray gas interactions $[9,10]$.

We propose a novel method in this paper to characterize the FEL x-ray pulse duration and temporal shape. A transverse $\mathrm{rf}$ deflector is used in conjunction with an e-beam energy spectrometer, located after the FEL undulator. By measuring the difference in the e-beam longitudinal phase space between FEL-on and FEL-off, we can obtain the time-resolved energy loss and energy spread induced from the FEL radiation, allowing the FEL $\mathrm{x}$-ray temporal shape to be reconstructed. This is a simple, single-shot method that is noninvasive to the FEL operation. The X-band transverse deflector that has been designed for the LCLS 
will cover the diagnostic range from a few fs to a few hundred fs over the full range of the FEL radiation wavelengths.

\section{RF DEFLECTOR AND SYSTEM LAYOUT}

The idea of using an rf deflecting structure (operating in the TM11 mode) to kick the electron beam was first proposed in the 1960s [11], and has been recently used for e-beam bunch length and temporal profile measurements in FELs and other accelerator facilities [12]. We assume the bunch is deflected in the horizontal plane by the high-frequency time variation of the deflecting fields, where the resulting horizontal beam width measured on a downstream screen (with a phase advance near $\pi / 2$ ) represents a single-shot measure of the absolute bunch temporal profile. This horizontally "streaked" e-beam is then sent to an energy spectrometer, which is composed of dipoles and quadrupoles providing large vertical momentum dispersion. If the optics is designed to have a vertical beta function small enough at the downstream screen, the vertical beam extent across the screen represents a momentum spread in the beam. With this setup, the e-beam longitudinal phase space dimensions (time and energy) are mapped into the real space transverse dimensions (horizontal and vertical).

In the FEL process, the interaction between an e-beam and an electromagnetic wave leads to e-beam energy modulation at the fundamental radiation wavelength. As electrons oscillate in the undulator, a periodic density modulation (the so-called "microbunching") at the radiation wavelength builds up. The microbunched e-beam then emits coherent radiation at the expense of the electron kinetic energy. The collective interaction of the beamradiation system leads to an exponential growth of the radiation intensity along the undulator distance. As a result, it causes electron energy loss and energy spread increase and the FEL power reaches saturation. At LCLS, the typical FEL-induced electron energy loss at saturation is more than $10 \mathrm{MeV}$ [1]. To obtain the $\mathrm{x}$-ray temporal profile, we first suppress the FEL process (e.g., by kicking e-beam to make a local oscillating orbit inside the undulator) and measure the e-beam time-energy phase space, from which we can get the e-beam temporal profile and also achieve a baseline (background) on the energy loss. To overcome the fluctuation of the measured background in the FEL-off case, multishot average and jitter correction should be considered in the practical measurements. Next, the FEL is restored and we measure the time-energy phase space again for each bunch. By subtracting the baseline measured with FEL-off, we can obtain the time-resolved energy loss or energy spread due to FEL radiation, shot by shot. The x-ray temporal power profile is then determined by combining the e-beam current profile and the timeresolved energy loss.

Figure 1 shows the beam line layout of this diagnostic system to be installed at the end of the LCLS main undulator. We use two 1-meter long X-band rf deflecting structures to provide a maximum horizontal kick of $46 \mathrm{MeV} / \mathrm{c}$, with $40 \mathrm{MW}$ input rf power at the deflecting structure [13].

\section{APPLICATION OF THE METHOD}

The deflecting force imparts a transverse momentum on the bunch with a small kick angle, $\Delta x^{\prime}$, given by

$$
\begin{aligned}
\Delta x^{\prime}(t) & =\frac{e V_{0}}{p c} \sin (2 \pi c t / \lambda+\varphi) \\
& \approx \frac{e V_{0}}{p c}\left(\frac{2 \pi}{\lambda} c t \cos \varphi+\sin \varphi\right),
\end{aligned}
$$

where $V_{0}$ is the deflector peak voltage, $p$ is the beam's longitudinal momentum in the structure, $\lambda=2.63 \mathrm{~cm}$ is the $\mathrm{rf}$ wavelength, $\varphi$ is the $\mathrm{rf}$ phase ( $=0$ at zero crossing), and $t$ is the electron time coordinate relative to the bunch center. The approximation is made that the bunch length is much shorter than the rf wavelength, $|c t| \ll \lambda / 2 \pi$.

From the deflecting point to the downstream screen, the beam is transported through a transfer matrix with angularto-spatial element $R_{12}=\left(\beta_{x d} \beta_{x s}\right)^{1 / 2} \sin \Delta \Psi$. Here $\beta_{x d}$ and $\beta_{x s}$ are the horizontal beta functions at the deflector and the screen, respectively, and $\Delta \Psi$ is the horizontal betatron phase advance from the deflector to the screen. The transverse position of each ultrarelativistic electron on the screen is then given:

$$
\Delta x(t)=\frac{e V_{0}}{p c} \sqrt{\beta_{x d} \beta_{x s}}|\sin \Delta \Psi|\left(\frac{2 \pi}{\lambda} c t \cos \varphi+\sin \varphi\right) .
$$

From Eq. (2) it is clear that, for bunch length measurement, operating at the zero-crossing phase $(\varphi=0)$ gives

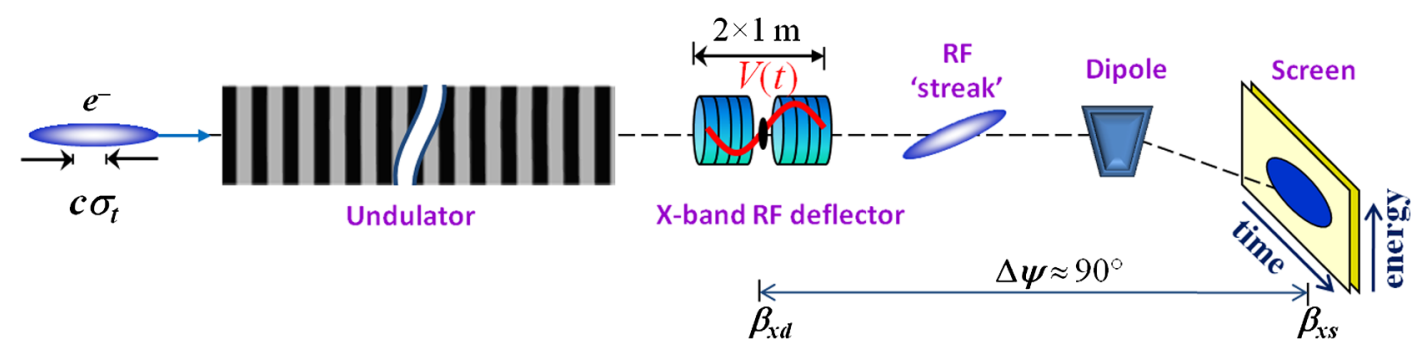

FIG. 1. A layout of the diagnostic system with a transverse rf deflector and an energy spectrometer. 
the best streaking effect with the horizontal beam size corresponding to the bunch length $\left(\sigma_{x} \propto c \sigma_{t}\right)$, while $\sin \varphi \neq 0$ gives a centroid offset which can be used for the calibration $(\langle\Delta x\rangle \propto \sin \varphi)$. For example, by measuring the horizontal centroid offset with a small rf phase shift around zero crossing, the size of horizontal dimension is calibrated relative to the absolute rf wavelength. From Eq. (2), near zero crossing, the time calibration factor can be written as

$$
S=\frac{\sigma_{x}}{c \sigma_{t}}=\frac{e V_{0}}{p c} \sqrt{\beta_{x d} \beta_{x s}}|\sin \Delta \Psi| \frac{2 \pi}{\lambda} .
$$

For the LCLS e-beam high-energy case of $14 \mathrm{GeV}$, based on the parameters listed in Table I, $S=128$. This means if the measured horizontal beam size $\sigma_{x}=128 \mu \mathrm{m}$, the actual bunch length is $c \sigma_{t}=1 \mu \mathrm{m}$.

Note that an X-band rf deflector has been chosen over an S-band one-like the original transverse deflector [11] —in order to impart a stronger sweep to the beam and thus improve the temporal resolution. At X-band the rf wavelength, $\lambda$, is smaller, giving a factor 4 improvement in Eq. (2). Furthermore, higher if gradients can be achieved at $\mathrm{X}$-band, allowing $V_{0}$ to be increased and further improving the gain in Eq. (2).

The vertical beam size measured after the vertically bent spectrometer represents the electron energy deviation, which is given by

$$
\Delta y=\eta_{y s} \delta,
$$

where $\eta_{y s}$ is the vertical momentum dispersion function at the screen, and $\delta$ is the relative energy deviation before the energy spectrometer. Note now we have a two-dimensional image on the screen with $x$ corresponding to time, and $y$ corresponding to energy.

Temporal resolution $\sigma_{t, r}$ and energy resolution $\sigma_{E, r}$ can be defined as

$$
\sigma_{t, r}=\frac{\sigma_{x 0}}{c S}, \quad \sigma_{E, r}=\frac{\sigma_{y 0}}{\eta_{y s}} E_{0}
$$

TABLE I. X-band transverse deflector parameters.

\begin{tabular}{lccc}
\hline \hline Parameter & Symbol & Value & Unit \\
\hline rf frequency & $f$ & 11.424 & $\mathrm{GHz}$ \\
Deflecting structure length & $L$ & $2 \times 1$ & $\mathrm{~m}$ \\
rf input power & $P$ & 40 & $\mathrm{MW}$ \\
Deflecting voltage (on crest) & $V_{0}$ & 48 & $\mathrm{MV}$ \\
Soft x-ray (e-beam 4.3 GeV) & & & \\
Calibration factor & $\mathrm{S}$ & 400 & \\
Temporal resolution (rms) & $\sigma_{t, r}$ & $\sim 1$ & $\mathrm{fs}$ \\
Energy resolution (rms) & $\sigma_{E, r}$ & 56 & $\mathrm{keV}$ \\
Hard x-ray (e-beam 14 GeV) & & & \\
Calibration factor & $\mathrm{S}$ & 128 & \\
Temporal resolution (rms) & $\sigma_{t, r}$ & $\sim 2$ & $\mathrm{fs}$ \\
Energy resolution (rms) & $\sigma_{E, r}$ & 100 & $\mathrm{keV}$ \\
\hline \hline
\end{tabular}

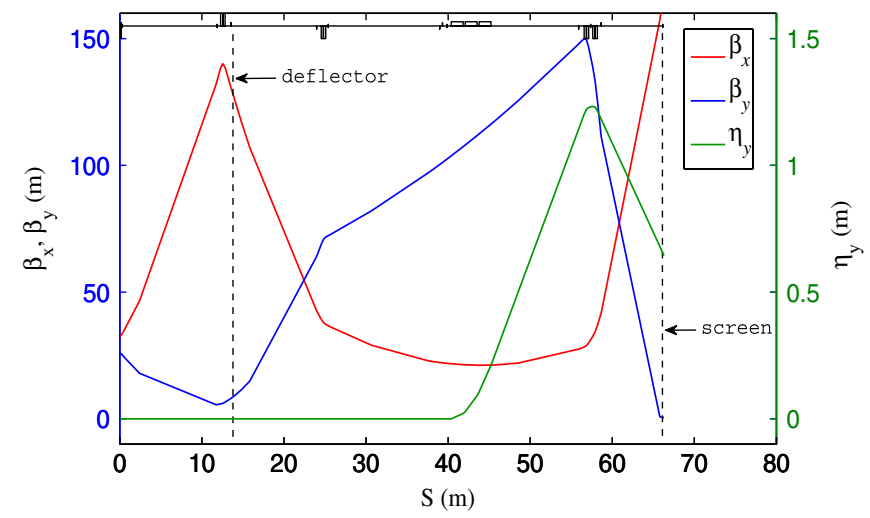

FIG. 2. The optics layout for this diagnostics. The locations of the deflector and screen are marked in the picture.

where $\sigma_{x 0}$ or $\sigma_{y 0}$ is the nominal transverse beam size at the screen (i.e., in the absence of deflecting voltage for $\sigma_{x 0}$, and in the absence of dispersion for $\sigma_{y 0}$ ), and $E_{0}$ is the average electron energy.

As seen from Eqs. (2), (4), and (5), the beam transportation optics should be optimized to achieve the best resolution. Larger horizontal beta function at the deflector and smaller vertical beta function at the screen are preferred. The optics beta and dispersion functions for the LCLS high-energy case $(14 \mathrm{GeV})$ are shown in Fig. 2, where the existing LCLS beam line magnets are used but their strengths have been adjusted to optimize the beta functions. In this example, the horizontal $\beta_{x d}=120 \mathrm{~m}$, $\beta_{x s}=170 \mathrm{~m}$, the phase advance $\Delta \Psi=90^{\circ}$, the vertical beta function at the screen $\beta_{y s}=0.54 \mathrm{~m}$, and the dispersive function $\eta_{y s}=0.65 \mathrm{~m}$. A very similar optics setup has also been achieved at low electron energy $(4.3 \mathrm{GeV})$ for soft x-ray generation.

The main parameters are summarized in Table I, based on a normalized projected emittance of $0.6 \mu \mathrm{m}$. The potential temporal resolution is $\sim 1 \mathrm{fs}$ rms for LCLS soft $\mathrm{x}$ rays, and $\sim 2 \mathrm{fs}$ rms for hard $\mathrm{x}$ rays.

\section{SIMULATION STUDIES}

Start-to-end simulations have been carried out to verify this scheme. IMPACT-T [14] and ELEGANT [15] codes have been used in the injector and main linac, including bunch compressors. In the undulator, a three-dimensional (3D) FEL simulation code GENESIS [16] has been adopted for FEL simulations, where the resistive wakefields from the undulator chamber and the spontaneous undulator radiation are also included. At the end of the undulator, the dumped particles are used again by ELEGANT to track them through the transverse deflector and the energy spectrometer down to the dump screen. From the simulated images with FEL-off and FEL-on on the dump screen, we can analyze the x-ray pulse duration.

We first show an example of the LCLS hard x-ray case (radiation wavelength of $1.5 \AA$, e-beam energy of 
13.6 GeV, total undulator length of $132 \mathrm{~m}$ including breaks) with a nominal operating charge of $250 \mathrm{pC}$. The average e-beam peak current is about $3 \mathrm{kA}$. Since the resistive wakefields in the linac rf structure lead to a third-order nonlinear curvature in the longitudinal phase space, we typically have a "double-horn" shape in the current profile. It is of great importance and interest to characterize the lasing process from this complicated bunch shape.

Figures 3(a) and 3(b) show the simulated "measurements" of the projected transverse images at the dump screen, with the horizontal axis representing time, and the vertical axis representing energy. Clearly, we can see the difference in the energy dimension between FEL-on and FEL-off. When the FEL is switched off [Fig. 3(a)], we measure a time-resolved e-beam energy $\left[E_{\mathrm{FEL} \text { off }}(t)\right]$ and energy spread $\left[\sigma_{E_{\mathrm{FEL} \text { off }}}(t)\right]$. The main collective effects include those from undulator chamber wakes, undulator spontaneous radiation, transverse deflecting, and coherent synchrotron radiation (CSR) in the spectrometer dipoles.

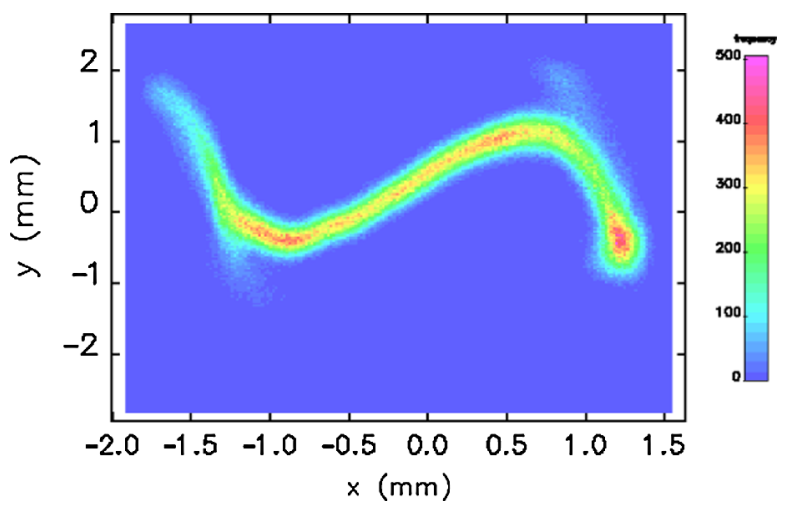

(a) FEL-off

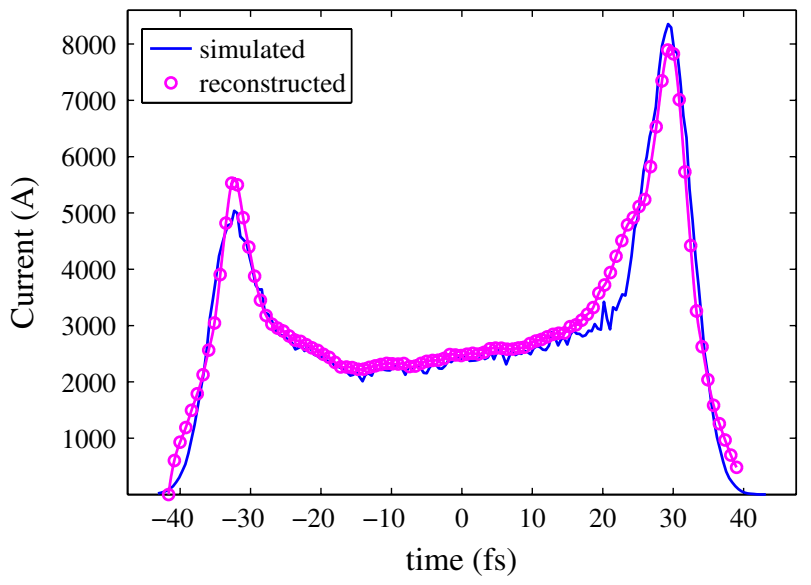

(c) e-beam current profile
When switched on [Fig. 3(b)], the FEL radiation results in an additional energy loss $\left[\Delta E_{\mathrm{FEL}}(t)\right]$ and energy spread $\left[\sigma_{E_{\mathrm{FEL}}}(t)\right]$. Then we measure the e-beam energy $\left(E_{\mathrm{FEL} \mathrm{on}}\right)$ and energy spread $\left(\sigma_{E_{\mathrm{FEL} \mathrm{on}}}\right)$ for the FEL-on case. From the two measurements we can determine the time-sliced energy loss or energy spread increase purely induced from the FEL radiation:

$$
\begin{aligned}
\Delta E_{\mathrm{FEL}}(t) & =E_{\mathrm{FEL} \mathrm{off}}(t)-E_{\mathrm{FEL} \text { on }}(t), \\
\sigma_{E_{\mathrm{FEL}}}(t) & =\sqrt{\sigma_{E_{\mathrm{FEL}}^{2} \text { on }}(t)-\sigma_{E_{\mathrm{FEL} \text { off }}}^{2}(t) .}
\end{aligned}
$$

The horizontal projection of the images in Figs. 3(a) and 3 (b) represents the e-beam temporal profile $I(t)$. The transverse deflector method provides an additional technique for eliminating systematic correlation errors. The upstream bunch compressors in the LCLS are in the horizontal plane and the CSR from their bends introduces a transverse kick to the electrons which is correlated to their longitudinal position in the bunch [17]. This correlation between the horizontal and longitudinal planes can affect the phase

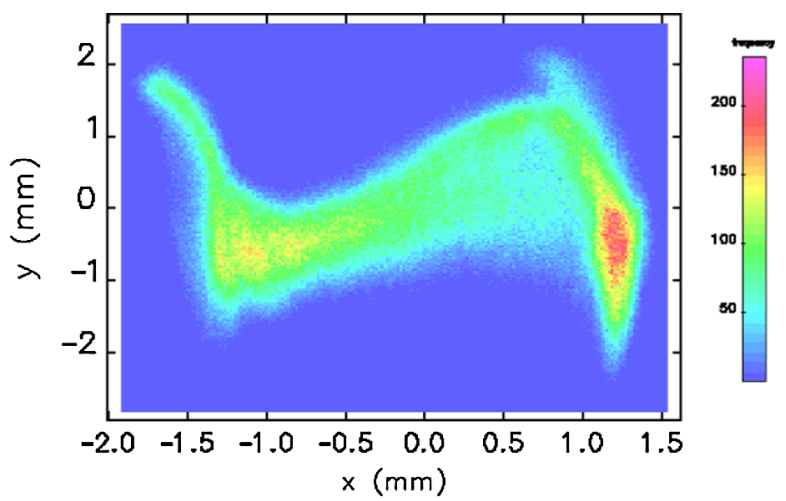

(b) FEL-on

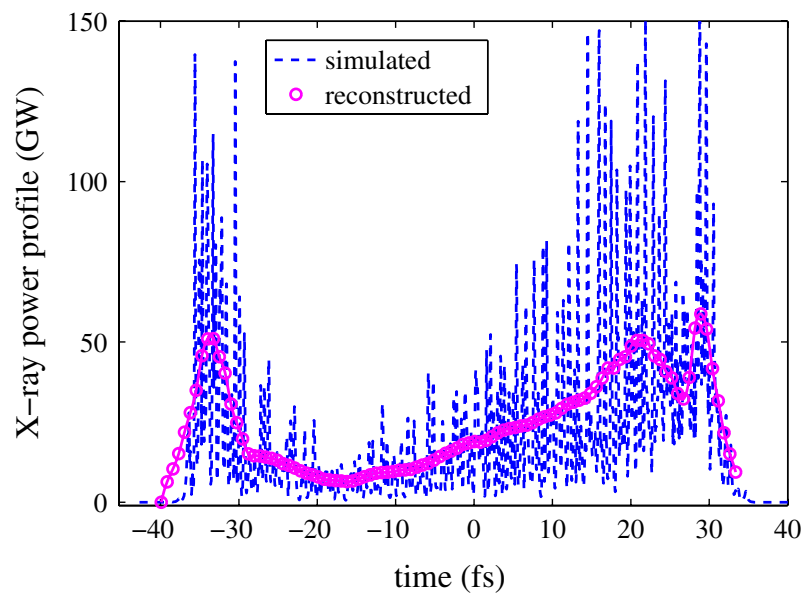

(d) $\mathrm{x}$-ray profile

FIG. 3. The simulated images on the screen representing e-beam longitudinal phase space for FEL-off (a) and FEL-on (b). The bunch charge is $250 \mathrm{pC}$ with an energy of $13.6 \mathrm{GeV}$. Parts (c) and (d) show the reconstructed e-beam current and FEL x-ray profiles (magenta) comparing with the simulated ones (blue). The bunch head is to the left. 
space reconstruction technique because the deflector also streaks the beam horizontally. This effect can be canceled by performing a second measurement at the other rf zerocrossing phase, $180^{\circ}$ from the first measurement [18]. An effective calibration factor can be defined after the dual rf zero-crossing measurements. Figure 3(c) shows the reconstructed e-beam current profile from two zero-crossing phases comparing with the original one.

With the obtained time-sliced energy loss and current, the $\mathrm{x}$-ray power profile is directly determined with an absolute power scale $\left[P(t)=\Delta E_{\mathrm{FEL}}(t) \times I(t)\right]$. The reconstructed $\mathrm{x}$-ray profile from the energy loss for this hard $\mathrm{x}$-ray example is shown in Fig. 3(d). Since LCLS is operating in the self-amplified spontaneous emission (SASE) mode, there are many longitudinal spikes whose typical width is $\sim 0.2 \mathrm{fs}$ in this hard $\mathrm{x}$-ray wavelength. The reconstructed pulse shape is a smooth approximation to the actual profile, where the finer spikes are smeared out due to the limited temporal resolution. Using the energy spread data we get a similar x-ray profile. To obtain the absolute power scale from the energy spread analysis it requires an additional measurement of the total $\mathrm{x}$-ray pulse energy.

Comparing the e-beam current profile with the x-ray profile shown in Fig. 3, we can see that the shape of the $\mathrm{x}$-ray profile deviates from the e-beam current profile, with less lasing right after the horn at the head of the bunch. This is the result of the wakefields in the undulator chamber suppressing the FEL lasing from the first horn at the head of the bunch.

Low charge operation mode with $20 \mathrm{pC}$ at LCLS has been used in many $\mathrm{x}$-ray user experiments for producing $\mathrm{x}$-ray pulses of a few fs [2]. Since these short x-ray pulses typically only have a few spikes, there is a large variation on the pulse shape. This makes the measurement of the actual x-ray pulse profile even more critical. We show an example of the soft $\mathrm{x}$-ray case right after saturation. The e-beam bunch charge is $20 \mathrm{pC}$ and the energy is $4.3 \mathrm{GeV}$. In the second bunch compressor, the e-beam is overcompressed so we can have a Gaussian-like current profile to generate a shorter $\mathrm{x}$-ray pulse [2]. The longitudinal phase space simulated at the dump screen is shown in Fig. 4. By

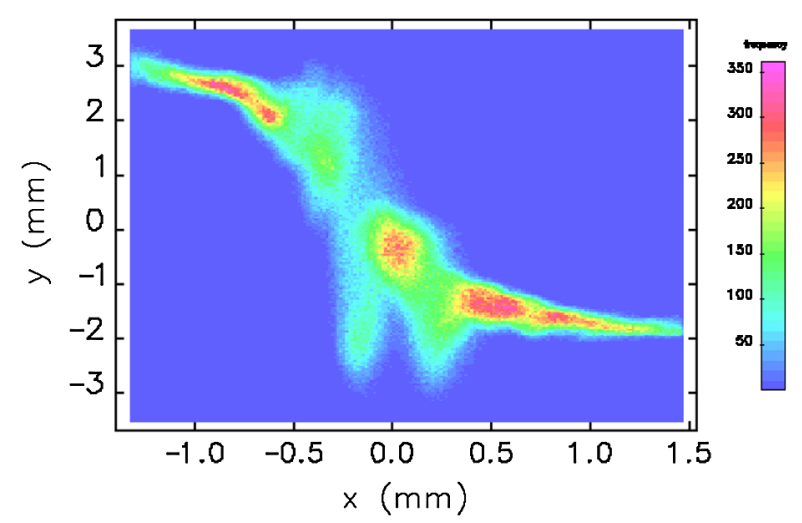

(b) FEL-on

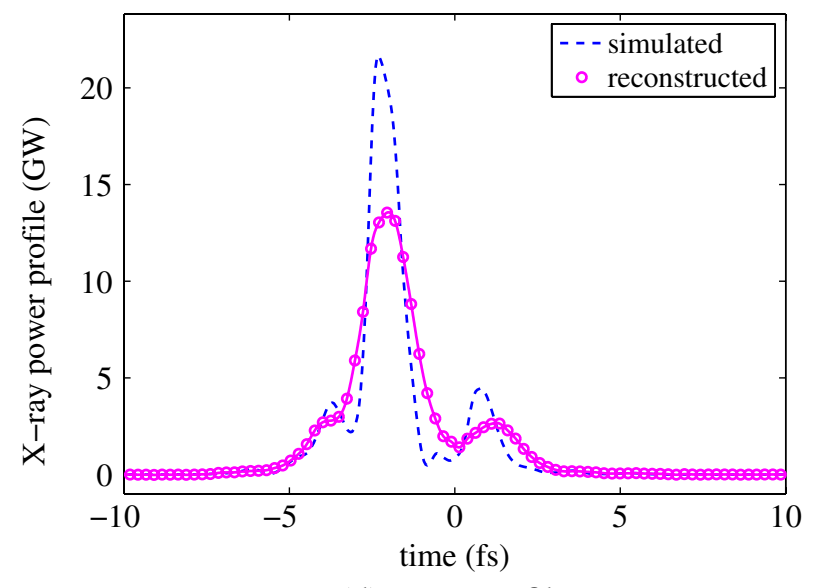

(d)x-ray profile

FIG. 4. The simulated images on the screen representing e-beam longitudinal phase space for FEL-off (a) and FEL-on (b). The bunch charge is $20 \mathrm{pC}$ with an energy of $4.3 \mathrm{GeV}$. Parts (c) and (d) show the reconstructed e-beam current and FEL x-ray profiles (magenta) comparing with the simulated ones (blue). The bunch head is to the left. 
subtracting the nonlasing background, the $\mathrm{x}$-ray profile is reconstructed [Fig. 4(d)]. The e-beam is about 4 fs FWHM, and the FEL profile in this snapshot has one main spike, and two small side spikes. Comparing the reconstructed $\mathrm{x}$-ray profile with the simulated one, we see some distortions in the profile peaks but still it is very encouraging. Running into the deep saturation regime, the slippage effect between FEL and e-beam may affect the shape of the reconstructed $\mathrm{x}$-ray power profile, especially for long-wavelength radiations. At the $\mathrm{x}$-ray wavelengths, this slippage effect after saturation is not a big problem.

\section{DISCUSSIONS AND SUMMARY}

As described earlier, we suppress the FEL lasing process and record the e-beam longitudinal phase space as a baseline, then compare the FEL-on case with the saved baseline image to analyze the FEL x-ray profile. In the simulation examples, we assume a stable e-beam without timing or energy jitter from shot to shot. The pulse-by-pulse jitter issues have to be considered during the real measurements. By choosing the $\mathrm{X}$-band rf deflector, an increased temporal resolution has been achieved, but the price to be paid is an increased sensitivity to phase jitter between the bunch arrival time and the X-band rf system. This must be minimized by designing tight $\mathrm{rf}$ phase tolerances into the system. The present achievable X-band rf phase stability could be $<0.1^{\circ}$, however, the measured LCLS arrival time jitter is $\sim 50 \mathrm{fs}$ rms [1]. This beam arrival time jitter makes the calibration very difficult. At LCLS, two phase cavities located after the undulator are used to measure the beam arrival time with an accuracy of $\sim 10 \mathrm{fs}$ rms [19]. These arrival time data measured from the phase cavities can be used to correct the timing jitter during the transverse deflector calibration measurements. Using these corrections combined with multishot averaging, we can achieve a reasonably good calibration within a useful rf phase range. We can also consider reducing the rf power during the calibration and then scale the calibration factor based on the measured rf power. During the phase space measurements, since we operate at the zero-crossing phase region where the rf amplitude versus the phase is quite linear, these rf phase jitter and beam arrival jitter do not affect the measurement, though a relatively large size screen should be considered for the system design. The e-beam pulse-bypulse energy jitter can also be corrected with the beam position monitors in the dogleg before the undulator. The transverse jitter out of the undulator is small and does not cause an additional effect on the measurement. Also note that the transverse deflector introduces additional correlation between the horizontal position and the energy. This effect has been included in the simulation examples in this paper, and it should be small since this energy spread can be subtracted in the analysis using Eq. (6).
In summary, we have shown that the proposed transverse rf deflector located after the FEL undulator has the potential to reconstruct the $\mathrm{x}$-ray temporal profiles with a very high resolution down to a few fs. This single-shot method is widely applicable to any radiation wavelength, SASE or seeded FEL mode, without interruption to user operation. This data can be delivered to the x-ray experiments in real time on a pulse-by-pulse basis. In addition, the e-beam bunch length and temporal profile are also obtained, providing a useful tool for a detailed study on the FEL lasing process.

\section{ACKNOWLEDGMENTS}

We thank G. Bowden, V. Dolgashev, J. Wang, J. Welch, and D. Xiang for helpful discussions. This work was supported by Department of Energy Contract No. DEAC02-76SF00515.

[1] P. Emma et al., Nat. Photon. 4, 641 (2010).

[2] Y. Ding et al., Phys. Rev. Lett. 102, 254801 (2009).

[3] Z. Huang et al., Phys. Rev. ST Accel. Beams 13, 092801 (2010).

[4] Z. Huang et al., in Proceedings of 2011 Particle Accelerator Conference, New York, USA (2011), p. 2459.

[5] U. Frühling et al., Nat. Photon. 3, 523 (2009).

[6] A. Maier (private communication).

[7] P. Catravas et al., Phys. Rev. Lett. 82, 5261 (1999).

[8] A. Lutman et al., in Proceedings of FEL2011, Shanghai, China (2011).

[9] G. Geloni et al., arXiv:1001.3544v1.

[10] S. Düsterer et al., New J. Phys. 13, 093024 (2011).

[11] G. A. Loew and O.H. Altenmueller, SLAC Report No. SLAC-PUB-135, 1965.

[12] For example, see R. Akre et al., in Proceedings of the 8th European Particle Accelerator Conference, Paris, 2002 (EPS-IGA and CERN, Geneva, 2002), p. 1882; Z. Huang et al., Phys. Rev. ST Accel. Beams 13, 020703 (2010); M. Röhrs et al., Phys. Rev. ST Accel. Beams 12, 050704 (2009); P. Musumeci et al., Phys. Rev. Lett. 106, 184801 (2011).

[13] J. Wang and S. Tantawi, in Proceedings of LINAC 08, Victoria, BC, Canada.

[14] J. Qiang et al., Phys. Rev. ST Accel. Beams 9, 044204 (2006).

[15] M. Borland, Advanced Photon Source Report No. LS-287, 2000.

[16] S. Reiche et al., Nucl. Instrum. Methods Phys. Res., Sect. A 429, 243 (1999).

[17] K. Bane et al., Phys. Rev. ST Accel. Beams 12, 030704 (2009).

[18] H. Loos et al., Proceedings of FEL2005, Stanford, CA (2005), p. 632.

[19] A. Brachmann et al., in Proceedings of the IPAC'10 Conference, Kyoto, Japan (ICR, Kyoto, 2010); see also Report No. SLAC-PUB-14234. 